\title{
SUR LE DÉRIVÉ DU CUBOÏDE EULÉRIEN
}

\author{
PAR \\ JEAN LAGRANGE
}

L'objet de cette note est de montrer que le dérivé d'un cuboïde eulérien n'est jamais parfait.

(1) Appelons cuboïde entier un cuboïde dont les arêtes et les diagonales des faces sont des entiers; appelons cuboïde parfait un cuboïde entier dont la diagonale intérieure est un entier. L'étude des cuboïdes entiers a suscité de nombreux travaux sans que ceux-ci aboutissent à la preuve de l'existence ou de l'inexistence d'un cuboïde parfait; le dernier travail en date est celui de Leech [1] et [2]. Spohn [4] montre qu'un cuboïde eulérien (i.e. d'arêtes $a\left(4 b^{2}-c^{2}\right)$, $b\left(4 a^{2}-c^{2}\right), 4 a b c$ avec $\left.a^{2}+b^{2}=c^{2}\right)$ ne peut être parfait. Soit un cuboïde entier d'arêtes $x, y, z$, on appelle cuboïde dérivé le cuboïde dont les arêtes sont proportionnelles à $y x, z x, x y$; il est clair qu'un tel cuboïde est entier. Spohn [5] tente de montrer que le dérivé d'un cuboïde eulérien ne peut être parfait, mais sa démonstration n'est pas complète.

(2) Le dérivé d'un cuboïde eulérien a pour arêtes:

$$
u=4 b c\left(4 a^{2}-c^{2}\right), \quad v=4 a c\left(4 b^{2}-c^{2}\right), \quad w=\left(4 b^{2}-c^{2}\right)\left(4 a^{2}-c^{2}\right)
$$

avec

$$
a^{2}+b^{2}=c^{2}
$$

On doit montrer que $u^{2}+v^{2}+w^{2}$ n'est jamais entier. On obtient facilement

$$
w=16 a^{2} b^{2}-3 c^{4}, \quad u^{2}+v^{2}=16 c^{8}
$$

Si le cuboïde est parfait, l'équation

$$
25 c^{8}-96 c^{4} a^{2} b^{2}+256 a^{4} b^{4}=z^{2}
$$

doit avoir une solution avec $a b \neq 0$. Posant $x=4 a b, y=c^{2}$ on obtient

$$
\left(x^{2}-5 y^{2}\right)^{2}+4 x^{2} y^{2}=z^{2}
$$

On doit montrer que cette équation n'a que la solution triviale $x y=0$.

(3) Plus généralement, nous allons montrer le

Reçu par les rédacteurs le 6 mars 1978. 
THÉORÈME. Si $p$ est un nombre premier congru à $5 \bmod 8$ tel que $p-1$ n'ait pas de diviseur premier congru a $1 \bmod 4$, alors l'équation diophantienne:

$$
\left(x^{2}-p y^{2}\right)^{2}+4 x^{2} y^{2}=z^{2}
$$

n'a que la solution triviale $x y=0$.

La démonstration utilise la méthode de Pocklington [3].

Toutes les lettres (à l'exception de $p$ ) représentent des entiers relatifs non nuls.

Soit $x, y$ une solution non triviale de (1), on peut toujours supposer que $(x, p y)=1$. Les entiers $x$ et $y$ ne peuvent être tous deux impairs car l'équation (1) s'écrirait

$$
\left(\frac{x^{2}-p y^{2}}{2}\right)^{2}+x^{2} y^{2}=\left(\frac{z}{2}\right)^{2}
$$

et $\left(x^{2}-p y^{2}\right) / 2$ serait congru à $0 \bmod 4$ ce qui exigerait $p=1 \bmod 8$. Les entiers $x$ et $y$ sont donc de parités différentes et la solution générale de (1) est

$$
x y=u v, \quad x^{2}-p y^{2}=u^{2}-v^{2}
$$

$u$ et $v$, premiers entre eux de parités différentes. La première équation de (2) a pour solution générale

$$
x=\alpha \beta, \quad y=\gamma \delta, \quad u=\alpha \gamma, \quad v=\beta \delta
$$

$\alpha, \beta, \gamma, \delta$ premiers entre eux deux à deux, un seul de ces entiers étant pair.

Portant ces valeurs dans la seconde équation de (2) on obtient:

$$
\beta^{2}\left(\alpha^{2}+\delta^{2}\right)=\gamma^{2}\left(\alpha^{2}+p \delta^{2}\right) .
$$

Par un calcul mod 8 on voit que c'est $\delta$ qui est pair. Comme $p-1$ n'a pas de diviseur premier congru à $1 \bmod 4, \alpha^{2}+\delta^{2}$ et $\alpha^{2}+p \delta^{2}$ sont premiers entre eux. D'où

$$
\alpha^{2}+p \delta^{2}=\beta^{2}, \quad \alpha^{2}+\delta^{2}=\gamma^{2}
$$

La première équation de (3) a pour solution générale

$$
\alpha=\lambda^{2}-p \mu^{2}, \quad \beta=\lambda^{2}+p \mu^{2}, \quad \delta=2 \lambda \mu
$$

$\lambda$ et $\mu$ entiers, premiers entre eux.

Portant ces valeurs dans la seconde équation de (3) on obtient

$$
\left(\lambda^{2}-p \mu^{2}\right)^{2}+4 \lambda^{2} \mu^{2}=\gamma^{2}
$$

d'où une autre solution de l'équation (1); on a

$$
0<|\lambda \mu|<|\delta| \leq|\alpha \beta \lambda \delta|=|x y|
$$

Le principe de descente permet de conclure. 


\section{RÉFÉRENCES}

1. J. Leech, The rational cuboid revisited, Amer. Math. Monthly, 84 (1977) 518-533.

2. J. Leech, Tables relating to rational cuboids, Math. Comp., (à paraitre).

3. H. C. Pocklington, Some Diophantine impossibilities, Proc. Cambridge Phil. Soc., 17 (1914) 110-118.

4. W. G. Spohn, On the integral cuboid, Amer. Math. Monthly, 79 (1972) 57-59.

5. W. G. Spohn, On the derived cuboid, Canad. Math. Bull. 17 (1974) 575-577.

DÉPARTEMENT DE MathÉmatiQues

FACULTÉ DES SCIENCES

Reims, France 